(2) Open Access Full Text Article

REVIEW

\title{
Intraperitoneal chemotherapy in ovarian cancer: a review of tolerance and efficacy
}

This article was published in the following Dove Press journal:

Cancer Management and Research

22 November 2012

Number of times this article has been viewed

\section{Daniel L Chan \\ David L Morris \\ Archana Rao \\ Terence C Chua}

Hepatobiliary and Surgical Oncology Unit, UNSW Department of Surgery, and the St George Clinical School, University of New South Wales, St George Hospital, Kogarah, NSW, Australia
Correspondence: Terence Chua Hepatobiliary and Surgical Oncology Unit, UNSW Department of Surgery, St George Hospital, Kogarah, NSW 2217, Australia Email terence.chua@unsw.edu.au
Purpose: To review the two main approaches of intraperitoneal (IP) chemotherapy delivery in ovarian cancer: postoperative adjuvant IP chemotherapy after cytoreductive surgery (CRS) and intraoperative hyperthermic intraperitoneal chemotherapy (HIPEC).

Methods: A literature search was conducted to identify studies that employed postoperative adjuvant IP chemotherapy after CRS or combined CRS and intraoperative HIPEC in patients with ovarian cancer. Data of interest included chemotherapy protocol, morbidity and mortality, and survival data.

Results: Three large randomized controlled trials comprising 707 patients with advanced ovarian cancer who received postoperative adjuvant IP chemotherapy were reviewed. Morbidity rate ranged from $56 \%$ to $94 \%$ in IP chemotherapy, and mortality rate ranged from $1 \%$ to $2 \%$. Median disease-free survival ranged from 24 to 28 months, and overall survival ranged from 49 to 66 months. Planned chemotherapy completion rates ranged from $42 \%$ to $71 \%$. Twentyfour nonrandomized studies that reported HIPEC comprised 1167 patients with both advanced and recurrent ovarian cancer. In patients with advanced ovarian cancer, mortality ranged from $0 \%$ to $5 \%$, minor morbidity ranged from $16 \%$ to $90 \%$, and major morbidity ranged from $0 \%$ to $40 \%$. Median disease-free survival ranged from 13 to 56 months, and overall survival ranged from 14 to 64 months. Survival at 5 years ranged from 35\% to $70 \%$. In patients with recurrent ovarian cancer, the mortality rate ranged from $0 \%$ to $10 \%$, minor morbidity ranged from $7 \%$ to $90 \%$, and major morbidity ranged from $0 \%$ to $49 \%$. Median disease-free survival ranged from 13 to 24 months and overall survival from 23 to 49 months. Survival at 5 years ranged from $12 \%$ to $54 \%$.

Conclusion: There is level-one evidence suggesting the benefit of postoperative adjuvant intraperitoneal chemotherapy for patients with advanced ovarian cancer after cytoreductive surgery, albeit catheter-related complications resulted after treatment discontinuation. Studies report the use of HIPEC predominantly in the setting of recurrent disease and have demonstrated encouraging results, which merits further investigation in future clinical trials.

Keywords: intraperitoneal chemotherapy, ovarian carcinoma, hyperthermic, intraoperative, cytoreductive surgery

\section{Introduction}

Ovarian cancer is the fifth leading cause of cancer death in females, with an estimated 22,280 women in the United States being diagnosed, accounting for 15,500 deaths in 2012. ${ }^{1}$ Epithelial ovarian cancer accounts for the majority of ovarian cancers $(>75 \%)$. The diagnosis is often delayed because of the nonspecific nature of its presenting symptoms, most commonly abdominal bloating and gastrointestinal disturbances. This insidious onset results in diagnosis at an advanced stage. The prevalence of advanced 
stage disease with peritoneal and distant metastasis (FIGO stage III/IV) is high, with the chance of cure low. ${ }^{2}$ The overall 5 -year survival rate of patients with ovarian cancer of all stages is $44 \%,{ }^{2}$ and it decreases to $<25 \%$ in patients with advanced disease. ${ }^{3}$

Ovarian cancer spreads through exfoliation of malignant cells into peritoneal fluid, disseminating along the abdominal and pelvic peritoneum, resulting in peritoneal metastases. This was previously regarded as a preterminal condition. ${ }^{4}$ Although ovarian cancer is often responsive to initial maximal cytoreductive surgery (CRS) and platinum-based chemotherapy, there remains a high rate of recurrence and poor long-term survival. Maximal cytoreductive surgical efforts against recurrent ovarian cancer have been shown to be independently associated with overall survival. ${ }^{5}$ Intraperitoneal (IP) chemotherapy, given by infusion of chemotherapeutic agents directly into the peritoneum, has been investigated by some groups and has demonstrated an improvement in overall and disease-free survival. ${ }^{6,7}$ Two forms of IP chemotherapy may be delivered: postoperative adjuvant IP chemotherapy delivered as adjuvant treatment after recovery from CRS, and intraperitoneal chemotherapy delivered as a heated chemoperfusate intraoperatively, known as hyperthermic intraperitoneal chemotherapy (HIPEC) ${ }^{8}$ Adjuvant IP chemotherapy has been evaluated in several randomized trials, and HIPEC has been demonstrated to be effective in the management of peritoneal dissemination of other malignancies, including colorectal cancer, ${ }^{9}$ pseudomyxoma peritonei, ${ }^{10}$ and peritoneal mesothelioma. ${ }^{11} \mathrm{~A}$ recent systematic review on the combination of CRS and HIPEC in ovarian cancer suggests potential benefits in disease-free and overall survival rates, with acceptable rates of morbidity and mortality. ${ }^{12}$ The conclusion from this systematic analysis was limited by the heterogeneity and small sample size of available studies at the time.

This review serves to describe the tolerance and efficacy of the two approaches to IP chemotherapy delivery.

\section{Methods}

A literature search was conducted on the EMBASE, Medline, and PubMed databases using combinations of the search terms "intraperitoneal," "chemotherapy," "hyperthermic," "intraoperative," and "ovarian cancer." The search was limited to the English language and to humans. Studies that employed postoperative adjuvant IP chemotherapy after CRS or combined CRS and HIPEC in patients with ovarian cancer, published from 1995 to 2011, were selected for review.
Data of interest included the two main types of intraperitoneal chemotherapy protocol: postoperative adjuvant IP chemotherapy or intraoperative HIPEC, the definition and percentage of optimal CRS and time, minor morbidity, major morbidity, disease free, and overall and longer-term survival data. Morbidity where defined included minor morbidity where complications were resolved with medical management and where no invasive intervention was required. Major morbidity was defined as complications where urgent definitive or invasive intervention, such as surgical, ICU admission, or radiological intervention, was required.

\section{Postoperative adjuvant intraperitoneal chemotherapy}

The concept of delivering chemotherapy directly to the tumor led to the use of intraperitoneal chemotherapy in ovarian cancer. In 1978, Dedrick et al ${ }^{13}$ reported that when ovarian tumors present on the peritoneum were exposed directly to chemotherapy drugs, it resulted in a higher intratumoral drug concentration than that achieved via the systemic route. Early clinical studies indicate that intraperitoneal chemotherapy delivery achieves a 10 to 20 times-higher tumor-chemotherapy dose than does the systemic delivery route. ${ }^{14}$

The support for combined postoperative intravenous (IV)/IP chemotherapy comes from eight randomized controlled trials, which were analyzed in a Cochrane review published in 2006. ${ }^{7}$ Three large randomized controlled trials that employed IP catheter delivery of chemotherapy were identified and are included in this review. ${ }^{6,15-17}$ The three trials included 707 patients who received IP chemotherapy; their characteristics are summarized in Table 1. All studies included only stage III ovarian cancer patients. The studies employed a combination of IV and IP chemotherapy, and this was compared to IV-only chemotherapy control arms. The common IP chemotherapy agent employed was cisplatin $\left(100 \mathrm{mg} / \mathrm{m}^{2}\right)$ delivered every three weeks over six cycles.

Completion rates of all cycles of IP chemotherapy ranged from $42 \%$ to $71 \%$, compared with $58 \%-86 \%$ in IV chemotherapy. This difference was attributed to high catheter-related complication rates, as well as to adverse hematological and gastrointestinal events. Major complications occurred in $69 \%-90 \%$ of IV chemotherapy patients and in $56 \%-94 \%$ of IP chemotherapy patients. Minor morbidity was not recorded. Mortality rates were similar, ranging from $1 \%$ to $2 \%$ in the IP group and from $0 \%$ to $2 \%$ in the IV group. Median disease-free survival in the IP chemotherapy patients ranged from 24 to 28 months, which was superior to the IV chemotherapy patients, whose median disease-free survival 


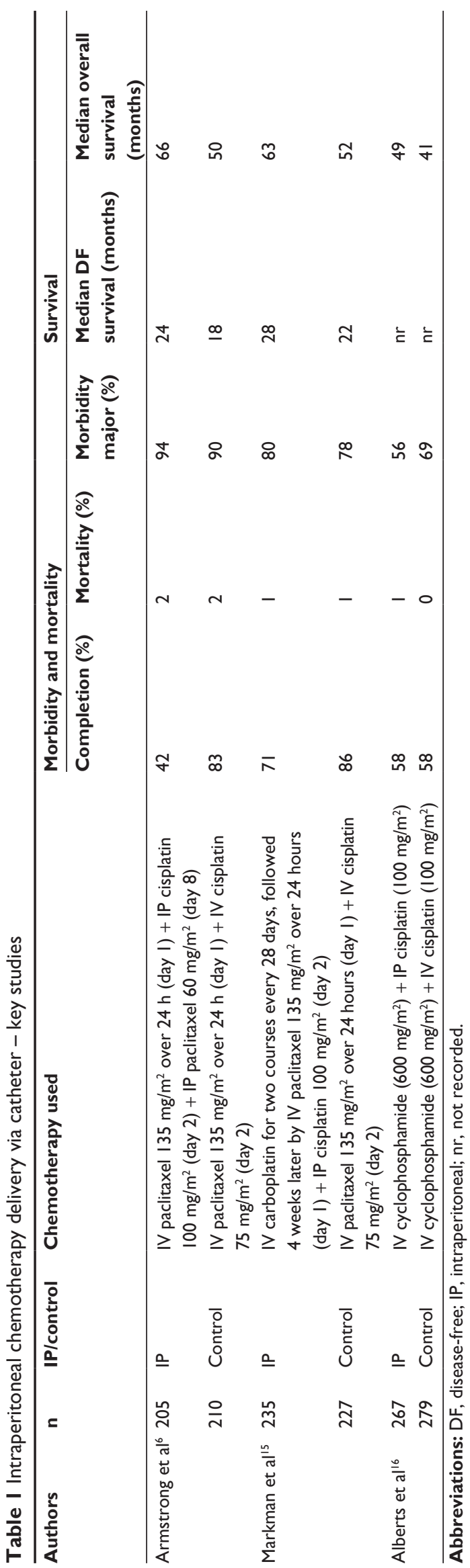

ranged between 11 and 22 months. This superior disease-free survival translated into overall survival gains, with the IP chemotherapy group and the IV chemotherapy group having a median overall survival of 49-66 and 41-52 months, respectively. Longer-term survival data at 3 and 5 years were not recorded. GOG-172 showed the longest median survival (65.6 months in the IP group) of all phase 3 GOG trials in advanced ovarian cancer. ${ }^{6}$

Intraperitoneal catheters are commonly implanted on the anterior abdominal wall after ovarian cancer cytoreduction or after full recovery from the initial surgery, and after thorough discussion and counseling about the potential benefits of this route of chemotherapy administration. IP chemotherapy delivery is selected for use in patients, following optimal cytoreduction. Although the recommended candidate is one who has not undergone a bowel resection, this is regarded as a relative contraindication, as this procedure should not be a limiting factor in precluding one's ability to achieve complete cytoreduction and subsequently receive this route of chemotherapy administration. IP chemotherapy may commence during the immediate early postoperative period, or once a patient recovers fully from ileus and has regained normal bowel function. Earlier administration may theoretically allow enhanced chemotherapy penetration into residual tumor nodules prior to the formation of the adhesions that often prevent free circulation of peritoneal chemoperfusate.

Despite the evidence from meta-analyses of randomized controlled trials demonstrating that IP chemotherapy achieves superior disease-free, overall survival, one of the factors limiting its widespread adoption is the associated toxicity, as demonstrated by the most recent randomized trial, GOG-172, ${ }^{6}$ where only $42 \%$ of patients in the IP arm completed their planned six cycles of IP chemotherapy. Walker et $\mathrm{al}^{17}$ examined the IP catheter outcomes in this trial. Of the 119 patients who did not complete the treatment, 16 patients (13\%) did not receive any IP chemotherapy, 68 patients $(57 \%)$ received one to two cycles of IP chemotherapy, and 35 patients (29\%) received three to five cycles of IP chemotherapy. In this group of patients, 40 of 119 patients (34\%) discontinued IP chemotherapy due to catheter-related problems, 45 patients (38\%) discontinued because of poor tolerance of the IP treatment, and 34 patients (29\%) discontinued because of chemotherapy complications or disease progression. ${ }^{17}$ This high rate of catheter and route-of-delivery issues raises doubts over the tolerability of IP chemotherapy; consequently, this approach has not become routine clinical practice despite the availability of level 1 evidence supporting its use. 


\section{Hyperthermic intraperitoneal chemotherapy (HIPEC)}

Hyperthermic intraperitoneal chemotherapy (HIPEC) was first described by Spratt et $\mathrm{al}^{18}$ in the treatment of peritoneal tumor from pseudomyxoma in 1980 . The rationale of combining heat with intraperitoneal chemotherapy is the added benefit of the synergistic effect of heat and cytotoxic drugs. ${ }^{19}$ Furthermore, this technique is delivered intraoperatively after cytoreduction, and this allows full peritoneal chemoperfusate circulation, with the timing of its administration to occur prior to the formation of adhesions that might limit peritoneal fluid circulation. This technique also avoids the need for implantation of peritoneal access devices, hence reducing catheter-related complications such as infection, and hence negating the issues of tolerance. ${ }^{20}$

Numerous nonrandomized comparative and observational studies employing a combination of CRS and HIPEC for ovarian cancer have been published. We identified 24 studies comprising 1167 patients. ${ }^{21-44}$ Eleven studies have previously been reviewed by our group, which we published as a systematic review encompassing 895 patients. The present review includes new data from an additional 418 patients. ${ }^{21-31} \mathrm{We}$ attempted to separate the disease timepoint of HIPEC treatment, as the majority of these studies report treating patients with both advanced and recurrent ovarian cancer without properly accounting for other contributing factors, such as platinum sensitivity and chemoresistance (Table 2). There are subtle variations in each institution's HIPEC protocol. The most common chemotherapy agent was cisplatin, which was used in 18 of the studies, either as monotherapy or in combination with mitomycin or doxorubicin. The median intra-abdominal temperature was $42^{\circ} \mathrm{C}$, with a range of $37^{\circ} \mathrm{C}-45^{\circ} \mathrm{C}$. The median duration of infusion was 90 minutes, with a range of 60-120 minutes. One study did not report its HIPEC protocol. ${ }^{26}$ The median duration of CRS and HIPEC was 480 minutes, with a range of 330-620 minutes. The majority of studies employed the definition of optimal cytoreduction as 0 or $<0.25 \mathrm{~cm}$, (range of $0 \mathrm{~cm}$ to $<2 \mathrm{~cm}$ ). Optimal cytoreduction was achieved in $66.3 \%$ of patients (range 19\%-100\%) (Table 3).

Fifteen studies reported data on 584 patients with advanced ovarian cancer undergoing HIPEC treatment. Collectively, these studies reported a perioperative mortality ranging from $0 \%$ to $5 \%$. Minor morbidity ranged from $16 \%$ to $90 \%$, and major morbidity ranged from $0 \%$ to $40 \%$, although only two studies had a major morbidity of $>20 \% .^{29,31}$ The median average of disease-free survival ranged from 13 to 56 months, and median overall survival from 24 to 64 months. Survival at 3 years was $48 \%-60 \%$, and at 5 years was $35 \%-70 \%$.

Table 2 HIPEC studies for ovarian cancer and patient background

\begin{tabular}{|c|c|c|c|c|c|}
\hline Authors & Level of evidence & $\mathbf{n}$ & Disease status & Chemoresistance & Previous chemo \\
\hline Tentes et $\mathrm{a}^{21}$ & Class III & 43 & Advanced and recurrent & Yes & Yes \\
\hline Königsrainer et $\mathrm{al}^{22}$ & Class III & 31 & Recurrent & Yes & Yes \\
\hline Fagotti et $\mathrm{a}^{23}$ & Class III & 41 & Recurrent & No & Yes \\
\hline Cascales Campos et $\mathrm{al}^{24}$ & Class III & 46 & Advanced and recurrent & $\mathrm{nr}$ & Yes \\
\hline Parson et $\mathrm{a}^{25}$ & Class III & 51 & Advanced & $\mathrm{nr}$ & Yes \\
\hline Spiliotis et $\mathrm{al}^{26}$ & Class II & 24 & Recurrent & $\mathrm{nr}$ & $\mathrm{nr}$ \\
\hline Deraco et $\mathrm{al}^{27}$ & Class III & 26 & Advanced & No & No \\
\hline Roviello et $\mathrm{a}^{28}$ & Class III & 53 & Advanced and recurrent & $\mathrm{nr}$ & Yes \\
\hline Pomel et $\mathrm{al}^{29}$ & Class III & 31 & Advanced & Yes & Yes \\
\hline Celeen et $\mathrm{a}^{30}$ & Class III & 42 & Recurrent & Yes & Yes \\
\hline Lim et $\mathrm{al}^{31}$ & Class III & 30 & Advanced & $\mathrm{nr}$ & Yes \\
\hline Bereder et $\mathrm{al}^{32}$ & Class III & 246 & Advanced and recurrent & Yes & Yes \\
\hline Pavlov et $\mathrm{a}^{33}$ & Class III & 56 & Advanced and recurrent & $\mathrm{nr}$ & Yes \\
\hline Guardiola et a $\left.\right|^{34}$ & Class III & 47 & Advanced & $\mathrm{nr}$ & Yes \\
\hline Di Giorgio et $\mathrm{al}^{35}$ & Class II & 47 & Advanced and recurrent & $\mathrm{nr}$ & Yes \\
\hline Bae et $\mathrm{al}^{36}$ & Class II & 67 & Advanced & No & Yes \\
\hline Cottee et $\mathrm{a}^{37}$ & Class III & 81 & Recurrent & Yes & Yes \\
\hline Raspagliesi et $\mathrm{al}^{38}$ & Class III & 40 & Recurrent & Yes & Yes \\
\hline Gori et al ${ }^{39}$ & Class III & 29 & Advanced & $\mathrm{nr}$ & Yes \\
\hline Look et $\mathrm{al}^{40}$ & Class III & 28 & Advanced & $\mathrm{nr}$ & Yes (18), no (10) \\
\hline Ryu et al ${ }^{41}$ & Class II & 57 & Advanced & No & Yes \\
\hline Zanon et $\mathrm{al}^{42}$ & Class III & 30 & Recurrent & $\mathrm{nr}$ & Yes \\
\hline Chatzigeorgiou et $\mathrm{al}^{43}$ & Class III & 20 & Recurrent & Yes & Yes \\
\hline Cavaliere et $\mathrm{al}^{44}$ & Class III & 20 & Recurrent & Yes & Yes \\
\hline
\end{tabular}

Abbreviation: $\mathrm{nr}$, not recorded. 


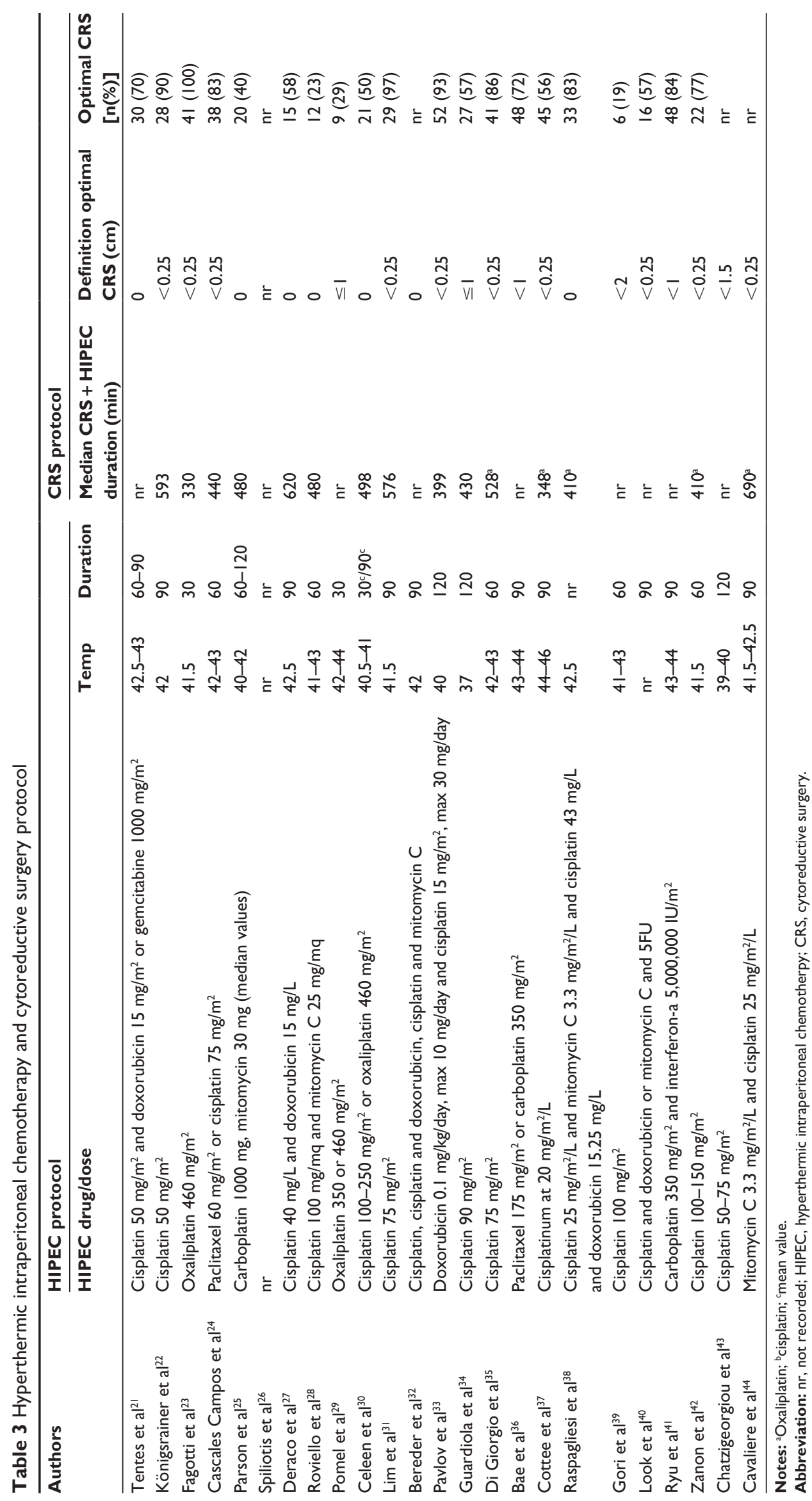


For patients with recurrent ovarian cancer, 13 studies included 583 patients. The perioperative mortality ranged from $0 \%$ to $10 \%$. Minor morbidity ranged from $7 \%$ to $90 \%$, and major morbidity ranged from $0 \%$ to $49 \%$. Median disease-free survival ranged from 13 to 24 months, and median overall survival from 23 to 49 months. Survival at 3 years was $35 \%-60 \%$, and at 5 years was $12 \%-54 \%$.

\section{Discussion}

The high rate of recurrence of ovarian cancer within the peritoneal cavity and the limited role of IV chemotherapy compared to intraperitoneal chemotherapy delivery after cytoreduction supports the role of intraperitoneal chemotherapy in achieving locoregional control within the peritoneum in ovarian cancer. There is a theoretical advantage in the delivery of high-concentration chemotherapeutic agents that act directly and eliminate residual microscopic disease, thereby achieving a pharmacokinetic profile of attaining a high drug concentration that enhances drug-tumor penetration.

The studies included in this review highlight the body of evidence supporting the advantages of intraperitoneal delivery of chemotherapy in combination with cytoreductive surgery in patients with advanced ovarian cancer. There is level 1 evidence that demonstrates the benefits of adjuvant postoperative IP chemotherapy in improving disease-free and overall survival. However, poor treatment tolerance has been the major inhibitor of the routine use of IP chemotherapy. ${ }^{45}$ The lack of uptake of IP chemotherapy into routine clinical practice, despite the published results of three major randomized trials, ${ }^{6,15,16}$ may also be a result of the different type of intraperitoneal chemotherapy (cisplatin), compared to the intravenous chemotherapy (carboplatin) that is being used. The 2004 International Gynecologic Cancer Intergroup Ovarian Cancer Consensus Conference ${ }^{45}$ recommends the use of intravenous carboplatin AUC 5-7.5 and paclitaxel $-175 \mathrm{mg} / \mathrm{m}^{2} / 3$ hours given every 3 weeks for six cycles as the standard of care in patients with advanced ovarian cancer. ${ }^{45}$ This recommendation follows from evidence for improved toxicity and tolerability profiles of chemotherapy combinations of carboplatin and paclitaxel over combinations containing cisplatin ${ }^{46,47}$ and cyclophosphamide. ${ }^{48}$ Further, Aletti et $\mathrm{al}^{49}$ attempted to translate research data from these randomized trials into routine clinical practice in accordance with best-practice evidence. However, in their single institution study, the investigators encountered challenges similar to those present in GOG-172, namely, the poor tolerability of IP chemotherapy that resulted only in $36 \%$ of patients completing the planned treatment. Their reasons for discontinuing treatment included catheterrelated complications (38\%), nephrotoxicity (14\%), and sepsis $(14 \%) .{ }^{49}$ Hence, although potential survival benefits may be obtained with IP chemotherapy, the morbidity of IP complications, the inability to complete planned treatment, and the possible effect on survival outcomes of unknown implications of not being able to complete treatment have limited clinicians' willingness to embrace IP chemotherapy as the standard chemotherapy delivery route.

Similar data also exists from nonrandomized studies of HIPEC; however, for this treatment to be considered for future routine practice, further commitment to sufficiently powered and well-designed randomized controlled trials is essential. $^{50,51}$

The Cochrane review of IP chemotherapy in women undergoing treatment for initial management of advanced ovarian cancer demonstrated a $21 \%$ decrease in the risk of death (HR 0.79, 95\% CI 0.70-0.90) in the patients undergoing combined IV/IP therapy, versus those undergoing IV therapy alone. ${ }^{7}$ The review of the literature regarding HIPEC (summarized in Tables 4 and 5) shows the median disease-free survival was 13-74 months in advanced, and 13-24 months in recurrent, ovarian cancer, in the studies reviewed. This compares favorably to IV chemotherapy delivered to platinum-sensitive disease (9-14 months) ${ }^{52,53}$ and platinum-resistant disease (13 months). ${ }^{54}$ We also found high 3-year and 5-year survival rates in both advanced and recurrent ovarian cancer. Fives studies demonstrated $>$ $50 \%$ survival at 5 years, in advanced cancer. The promising improvement in survival outcomes may be related to the development of high-volume specialized institutions. ${ }^{55}$

The optimal choice of chemotherapeutic agent in HIPEC is unclear and is probably based on extrapolation of evidence from the efficacy of intravenous chemotherapy. Theoretically, the selected agent should be water-soluble, and have a low peritoneal clearance, high peritoneal concentration, high systemic clearance, and enhanced penetration and cytotoxic ability with hyperthermic application. The majority of HIPEC studies on ovarian cancer have used IP cisplatin, ${ }^{21,22,24,27,28,30-35,37-40,42-44}$ as the HIPEC agent. Other studies included in this review employed doxorubicin, $21,27,32,33,38,40$ mitomycin $C,{ }^{24,28,32,38,40,44}$ oxaliplatin, ${ }^{23,29,30}$ paclitaxel, ${ }^{24,36}$ and gemcitabine. ${ }^{21}$

The adoption of both postoperative adjuvant IP chemotherapy and HIPEC into routine practice is potentially limited by concerns over tolerability and morbidity. Cytoreductive surgery and HIPEC for advanced $(0 \%-5 \%)$ and recurrent 


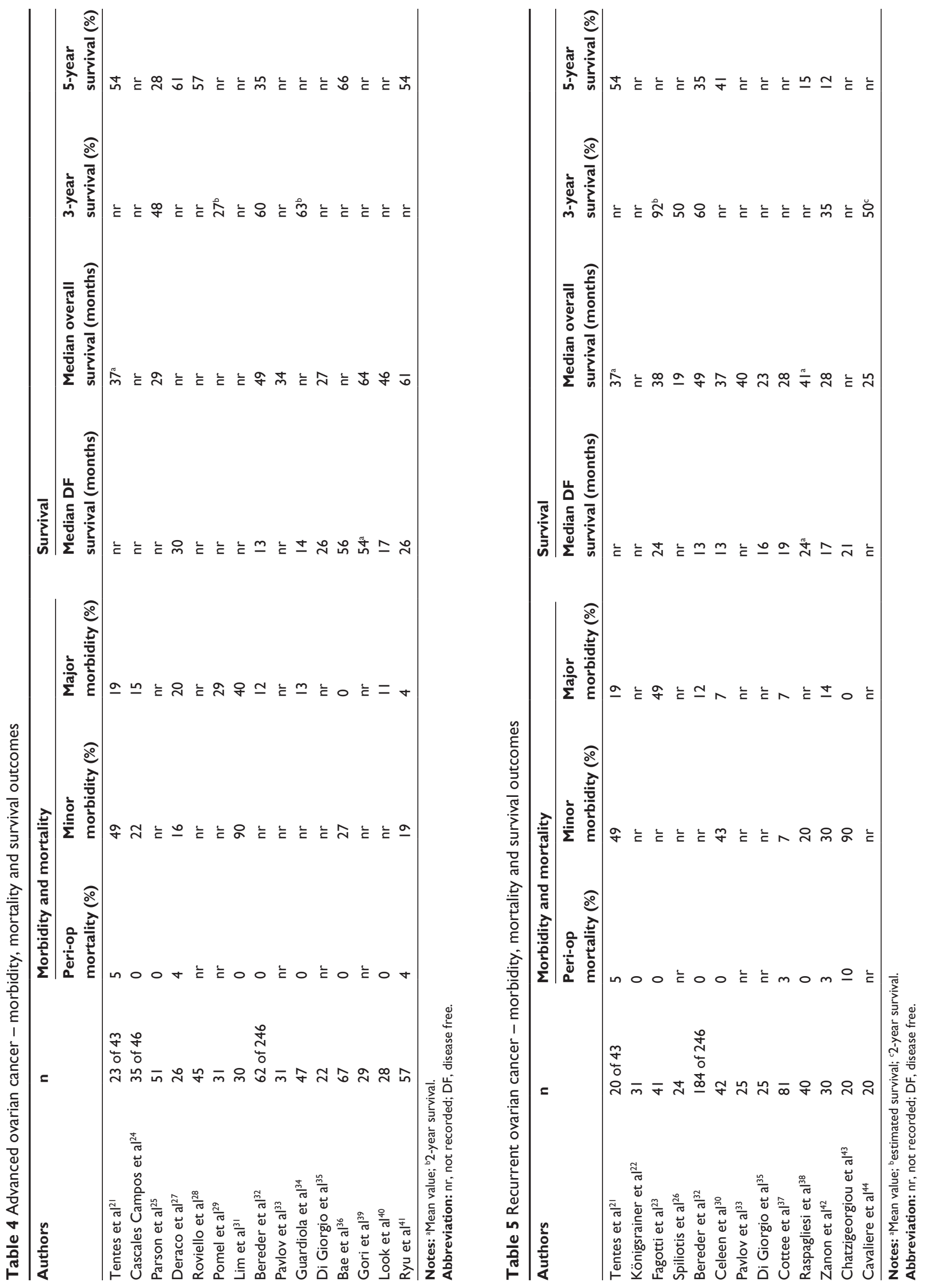


$(0 \%-10 \%)$ ovarian cancer mortality rates were consistent with previous high-volume tertiary institutional evidence and similar to mortality rates of other major gastrointestinal surgery. ${ }^{55}$ Although mortality rates for HIPEC for advanced ovarian cancer were slightly higher than rates in the postoperative adjuvant IP chemotherapy treatment cohort $(0 \%-2 \%)$ for which only the complications arising from the six cycles of treatment have been reported, it is important to take into consideration that the high complication rate reported for CRS HIPEC combines the complication rates from both the surgical and chemotherapy components of the treatment. The higher morbidity and mortality observed with recurrent disease may be related to patients having previously undergone radical surgery to achieve complete primary cytoreduction, as recommended by a recent Cochrane review. ${ }^{56}$ Perhaps the promising combination of CRS and HIPEC in the primary setting of advanced ovarian cancer should be explored further.

The postoperative adjuvant IP chemotherapy completion rate was as low as $42 \%$ in GOG- $172 .{ }^{6}$ Catheter-related complications were the primary reason for discontinuation in $34 \%$ of patients. ${ }^{17}$ This suggests the catheter choice and timing of insertion requires further investigation. Survival benefits were achieved despite low rates of treatment completion, which suggests adjuvant IP chemotherapy still had a significant role.

Adjuvant postoperative IP chemotherapy in the three randomized controlled trials was employed in primary advanced stage III ovarian cancer only in conjunction with optimal CRS debulking. The effect of IP chemotherapy in stage IV and recurrent disease where optimal CRS might not necessarily be achieved is under investigation. ${ }^{57}$ The role of other chemotherapeutic agents beyond cisplatin is also being explored by current trials. ${ }^{58}$

The potential benefits of HIPEC compared to postoperative IP chemotherapy relate to the theoretical advantages of its synergistic hyperthermic effect on chemotherapy, to its delivery of chemotherapy to peritoneal surfaces before the development of adhesions, and to the potential it creates for avoiding postoperative catheter-related complications and subsequently improving the ability to effectively deliver intraperitoneal chemotherapy. These advantages, combined with the evidence from observational and nonrandomized data, provide a strong rationale for undertaking further clinical trial investigations in this area. However, our review of the data regarding HIPEC also highlights the significant incidence of morbidity associated with the treatment, with major morbidity ranging from $0 \%$ to $40 \%$ (only two studies had a major morbidity rate of $>20 \%$ ). In one study, $40 \%$ of patients required invasive medical intervention, but reported no surgical or intensive-care unit intervention. ${ }^{31}$ Another study was discontinued due to high rates of major morbidity, with $29 \%$ of patients requiring reoperation for intra-abdominal bleeding. ${ }^{29}$ This was the only study that employed oxaliplatin as the sole chemotherapeutic agent. The increased rates of bleeding might have been due to the greater hematological toxicity of oxaliplatin, as compared with other chemotherapeutic agents, as demonstrated in its use in colorectal and appendiceal tumors. ${ }^{59}$

Current evidence establishes the role of postoperative IP chemotherapy in the adjuvant setting, in patients with advanced ovarian cancer who have undergone optimal cytoreductive surgery. Yet it leaves questions unanswered with regard to the optimal IP chemotherapy regimen in terms of tolerability, the role of HIPEC, and the role of IP chemotherapy in the setting of recurrence. Despite evidence suggesting the efficacy of CRS and HIPEC in patients with both advanced and recurrent ovarian cancer, the nonrandomized nature of the data and its heterogeneity (both in terms of patient selection and treatment protocols) make it difficult to make direct comparisons with randomized data from trials of postoperative adjuvant IP chemotherapy. This difficulty again highlights the need for further prospective randomized trials to identify the potential role of HIPEC in ovarian cancer. Such trials would establish whether HIPEC presents an acceptable alternative to current standards of care for the adjuvant treatment of advanced ovarian cancer, including postoperative IP chemotherapy, and whether IP chemotherapy offers a new dimension to the multimodality approach in managing recurrent ovarian cancer.

\section{Disclosure}

The authors report no conflicts of interest in this work.

\section{References}

1. Seigal R, Naishadham D, Jemal A. Cancer statistics. CA Cancer J Clin. 2012;62(1):10-29.

2. Goff BA, Mandel L, Muntz HG, Melancon $\mathrm{CH}$. ovarian carcinoma diagnosis. Cancer. 2000;89(10):2068-2075.

3. Ozols RF. Treatment goals in ovarian cancer. Int J Gynecol Cancer. 2005;15(Suppl 1):S3-S11.

4. Sugarbaker PH. Peritoneal Carcinomatosis Principles of Management. 1st ed. Boston: Kluwer; 1996.

5. Bristow RE, Puri I, Chi DS. Cytoreductive surgery for recurrent ovarian cancer: a meta-analysis. Gynecol Oncol. 2009;112(1):265-274.

6. Armstrong DK, Bundy B, Wenzel L, et al. Intraperitoneal cisplatin and paclitaxel in ovarian cancer. $N$ Engl J Med. 2006;354(1):34-43.

7. Jaaback $\mathrm{K}$, Johnson $\mathrm{N}$, Lawrie TA. Intraperitoneal chemotherapy for the initial management of primary epithelial ovarian cancer [review]. Cochrane Database Syst Rev. 2011;11:CD005340. 
8. Witkamp AJ, de Bree E, Van Goethem R, Zoetmulder FA. Rationale and techniques of intra-operative hyperthermic intraperitoneal chemotherapy [review]. Cancer Treat Rev. 2001;27(6):365-374.

9. Yan TD, Black D, Savady R, Sugarbaker PH. Systematic review on the efficacy of cytoreductive surgery combined with perioperative intraperitoneal chemotherapy for peritoneal carcinomatosis from colorectal carcinoma. J Clin Oncol. 2006;24(24):4011-4019.

10. Yan TD, Black D, Savady R, Sugarbaker PH. A systematic review on the efficacy of cytoreductive surgery and perioperative intraperitoneal chemotherapy for pseudomyxoma peritonei. Ann Surg Oncol. 2007;14(2): 484-492.

11. Yan TD, Welch L, Black D, Sugarbaker PH. A systematic review on the efficacy of cytoreductive surgery combined with perioperative intraperitoneal chemotherapy for diffuse malignancy peritoneal mesothelioma. Ann Oncol. 2007;18(5):827-834.

12. Chua TC, Robertson G, Liauw W, Farrell R, Yan TD, Morris DL. Intraoperative hyperthermic intraperitoneal chemotherapy after cytoreductive surgery in ovarian cancer peritoneal carcinomatosis: systematic review of current results. J Cancer Res Clin Oncol. 2009;135(12): 1637-1645.

13. Dedrick RL, Myers CE, Bungay PM, DeVita VT Jr. Pharmacokinetic rationale for peritoneal drug administration in the treatment of ovarian cancer. Cancer Treat Rep. 1978;62(1):1-11.

14. Francis P, Rowinsky E, Schneider J, Hakes T, Hoskins W, Markman M. Phase I feasibility and pharmacologic study of weekly intraperitoneal paclitaxel: a Gynecologic Oncology Group pilot study. J Clin Oncol. 1995;13(12):2961-2967.

15. Markman MB, Bundy BN, Alberts DS, et al. Phase III trial of standarddose intravenous cisplatin plus paclitaxel versus moderately high-dose carboplatin followed by intravenous paclitaxel and intraperitoneal cisplatin in small-volume stage III ovarian carcinoma: an intergroup study of the Gynecologic Oncology Group, Southwestern Oncology Group, and Eastern Cooperative Oncology Group. J Clin Oncol. 2001;19(4): 1001-1007.

16. Alberts DS, Liu PY, Hannigan EV, et al. Intraperitoneal cisplatin plus intravenous cyclophosphamide versus intravenous cisplatin plus intravenous cyclophosphamide for stage III ovarian cancer. $N$ Engl J Med. 1996;335(26):1950-1955.

17. Walker JL, Armstrong DK, Huang HQ, et al. Intraperitoneal catheter outcomes in a phase III trial of intravenous versus intraperitoneal chemotherapy in optimal stage III ovarian and primary peritoneal cancer: a Gynecologic Oncology Group study. Gynecol Oncol. 2006;100(1):27-32.

18. Spratt JS, Adcock RA, Muskovin M, Sherrill W, McKeown J. Clinical delivery system for intraperitoneal hyperthermic chemotherapy. Cancer Res. 1980;40(2):256-260.

19. Kusumoto T, Holden SA, Ara G, Teicher BA. Hyperthermia and platinum complexes: time between treatments and synergy in vitro and in vivo. Int J Hyperthermia. 1995;11(4):575-586.

20. Sugarbaker PH. Laboratory and clinical basis for hyperthermia as a component of intracavitary chemotherapy. Int J Hyperthermia. 2007;23(5): 431-442.

21. Tentes AA, Kakolyris S, Kyziridis D, Karamveri C. Cytoreductive surgery combined with hyperthermic intraperitoneal intraoperative chemotherapy in the treatment of advanced epithelial ovarian cancer. J Oncol. 2012;2012:358-341.

22. Konigsrainer I, Beckert S, Becker S, et al. Cytoreductive surgery and HIPEC in peritoneal recurrent ovarian cancer: experience and lessons learned. Langenbecks Arch Surg. 2011;396(7):1077-1081.

23. Fagotti A, Costantini B, Vizzielli G, et al. HIPEC in recurrent ovarian cancer patients: morbidity-related treatment and long-term analysis of clinical outcome. Gynecol Oncol. 2011;122(2):221-225.

24. Cascales Campos PA, Gil Martinez J, Galindo Fernandez PJ, et al. Perioperative fast track program in intraoperative hyperthermic intraperitoneal chemotherapy (HIPEC) after cytoreductive surgery in advanced ovarian cancer. Eur J Surg Oncol. Jun 2011;37(6): $543-548$
25. Parson EN, Lentz S, Russell G, Shen P, Levine EA, Stewart JH. Outcomes after cytoreductive surgery and hyperthermic intraperitoneal chemotherapy for peritoneal surface dissemination from ovarian neoplasms. Am J Surg. 2011;202(4):481-486.

26. Spiliotis J, Vaxevanidou A, Sergouniotis F, Lambropoulou E, Datsis A, Christopoulou A. The role of cytoreductive surgery and hyperthermic intraperitoneal chemotherapy in the management of recurrent advanced ovarian cancer: a prospective study [abstract]. J BUON. 2011;16(1): 74-79.

27. Deraco M, Kusamura S, Virzi S, et al. Cytoreductive surgery and hyperthermic intraperitoneal chemotherapy as upfront therapy for advanced epithelial ovarian cancer: multi-institutional phase-II trial. Gynecol Oncol. 2011;122(2):215-220.

28. Roviello F, Pinto E, Corso G, et al. Safety and potential benefit of hyperthermic intraperitoneal chemotherapy (HIPEC) in peritoneal carcinomatosis from primary or recurrent ovarian cancer. J Surg Oncol. 2010;102(6):663-670.

29. Pomel C, Ferron G, Lorimier G, et al. Hyperthermic intra-peritoneal chemotherapy using oxaliplatin as consolidation therapy for advanced epithelial ovarian carcinoma. Results of a phase II prospective multicentre trial. CHIPOVAC study. Eur J Surg Oncol. 2010;36(6):589-593.

30. Ceelen WP, Van Nieuwenhove Y, Van Belle S, Denys H, Pattyn P. Cytoreduction and hyperthermic intraperitoneal chemoperfusion in women with heavily pretreated recurrent ovarian cancer. Ann Surg Oncol. 2009;19(7):2352-2359.

31. Lim MC, Kang S, Choi J, et al. Hyperthermic intraperitoneal chemotherapy after extensive cytoreductive surgery in patients with primary advanced epithelial ovarian cancer: interim analysis of a phase II study. Ann Surg Oncol. 2009;16(4):993-1000.

32. Bereder J, Glehen O, Habre J, et al. Cytoreductive surgery combined with perioperative intraperitoneal chemotherapy for the management of peritoneal carcinomatosis from ovarian cancer: a multiinstitutional study of 246 patients. J Clin Oncol. 2009;27(Suppl 15):Abstract 5542.

33. Pavlov MJ, Kovacevic PA, Ceranic MS, Stamenkovic AB, Ivanovic AM, Kecmanovic DM. Cytoreductive surgery and modified heated intraoperative intraperitoneal chemotherapy (HIPEC) for advanced and recurrent ovarian cancer - 12-year single center experience. Eur J Surg Oncol. 2009;35(11):1186-1191.

34. Guardiola E, Delroeux D, Heyd B, et al. Intra-operative intra-peritoneal chemotherapy with cisplatin in patients with peritoneal carcinomatosis of ovarian cancer. World J Surg Oncol. 2009;7:14.

35. Di Giorgio A, Naticchioni E, Biacchi D, et al. Cytoreductive surgery (peritonectomy procedures) combined with hyperthermic intraperitoneal chemotherapy (HIPEC) in the treatment of diffuse peritoneal carcinomatosis from ovarian cancer. Cancer. 2008;113(2):315-325.

36. Bae JH, Lee JM, Ryu KS, et al. Treatment of ovarian cancer with paclitaxel- or carboplatin-based intraperitoneal hyperthermic chemotherapy during secondary surgery. Gynecol Oncol. 2007;106(1): 193-200.

37. Cotte E, Glehen O, Mohamed F, et al. Cytoreductive surgery and intraperitoneal chemo-hyperthermia for chemo-resistant and recurrent advanced epithelial ovarian cancer: prospective study of 81 patients. World J Surg. 2007;31(9):1813-1820.

38. Raspagliesi F, Kusamura S, Campos Torres JC, et al. Cytoreduction combined with intraperitoneal hyperthermic perfusion chemotherapy in advanced/recurrent ovarian cancer patients: The experience of National Cancer Institute of Milan. Eur J Surg Oncol. 2006;32(6): 671-675.

39. Gori J, Canstaño R, Toziano M, et al. Intraperitoneal hyperthermic chemotherapy in ovarian cancer. Int J Gynecol Cancer. 2005;15(2):233-239.

40. Look M, Chang D, Sugarbaker PH. Long-term results of cytoreductive surgery for advanced and recurrent epithelial ovarian cancers and papillary serous carcinoma of the peritoneum. Int $J$ Gynecol Cancer. 2004;14(1):35-41.

41. Ryu KS, Kim JH, Ko HS, et al. Effects of intraperitoneal hyperthermic chemotherapy in ovarian cancer. Gynecol Oncol. 2004;94(2): 325-332. 
42. Zanon C, Clara R, Chiappino I, et al. Cytoreductive surgery and intraperitoneal chemohyperthermia for recurrent peritoneal carcinomatosis from ovarian cancer. World J Surg. 2004;28(10):1040-1045.

43. Chatzigeorgiou K, Economou S, Chrysafis G, et al. Treatment of recurrent epithelial ovarian cancer with secondary cytoreduction and continuous intraoperative intraperitoneal hyperthermic chemoperfusion (CIIPHCP). Zentralbl Gynakol. 2003;125(10):424-429.

44. Cavaliere F, Perri P, Di Filippo F, et al. Treatment of peritoneal carcinomatosis with intent to cure. J Surg Oncol. 2000;74(1):41-44.

45. du Bois A, Quinn M, Thigpen T, et al. 2004 consensus statements on the management of ovarian cancer: final document of the 3rd International Gynecologic Cancer Intergroup Ovarian Cancer Consensus Conference (GCIG OCCC 2004). Ann Oncol. 2005;16(Suppl 8):S7-S12.

46. du Bois A, Lück HJ, Meier W, et al; for Arbeitsgemeinschaft Gynäkologische Onkologie Ovarian Cancer Study Group. A randomized clinical trial of cisplatin/paclitaxel versus carboplatin/paclitaxel as first-line treatment of ovarian cancer. J Natl Cancer Inst. 2003;95(17):1320-1329.

47. Ozols RF, Bundy BN, Greer BE, et al; for Gynecologic Oncology Group. Phase III trial of carboplatin and paclitaxel compared with cisplatin and paclitaxel in patients with optimally resected stage III ovarian cancer: a Gynecologic Oncology Group study. J Clin Oncol. 2003;21(17): 3194-3200.

48. McGuire WP, Hoskins WJ, Brady MF, et al. Cyclophosphamide and cisplatin compared with paclitaxel and cisplatin in patients with stage III and stage IV ovarian cancer. $N$ Engl J Med. 1996;334(1):1-6.

49. Aletti GD, Nordquist D, Harmann L, Gallenberg M, Long HJ, Cliby WA. From randomized trial to practice: single institution experience using the GOG 172 i.p. chemotherapy regimen for ovarian cancer. Ann Oncol. 2010; 21(9):1772-1778.

50. Chua TC, Helm CW, Robertson G, Morris DL. Establishing evidence for change in ovarian cancer surgery - proposing clinical trials of cytoreductive surgery and hyperthermic intraperitoneal chemotherapy (HIPEC) in ovarian cancer peritoneal carcinomatosis. Gynecol Oncol. 2009;115(1):166-168.
51. de Bree E, Helm CW. Hyperthermic intraperitoneal chemotherapy in ovarian cancer: rationale and clinical data. Expert Rev Anticancer Ther. 2012;12(7):895-911.

52. Pfisterer J, Plante M, Vergote I, et al. Gemcitabine plus carboplatin compared with carboplatin in patients with platinum-sensitive recurrent ovarian cancer: an intergroup trial of the AGO-OVAR, the NCIC CTG, and the EORTC GCG. J Clin Oncol. 2006;24(29):4699-4707.

53. Ferrandina G, Ludovisi M, De Vincenzo R, et al. Docetaxel and oxaliplatin in the second-line treatment of platinum-sensitive recurrent ovarian cancer: a phase II study. Ann Oncol. 2007;18(8):1348-1353.

54. Aravantinos G, Bafaloukos D, Fountzilas G, et al. Phase II study of docetaxel - vinorelbine in platinum-resistant, paclitaxel-pretreated ovarian cancer. Ann Oncol. 2003;14(7):1094-1099.

55. Chua TC, Yan TD, Saxena A, Morris DL. Should the treatment of peritoneal carcinomatosis by cytoreductive surgery and hyperthermic intraperitoneal chemotherapy still be regarded as a highly morbid procedure?: a systematic review of morbidity and mortality. Ann Surg. 2009;249(6):900-907.

56. Elattar A, Bafaloukos D, Winter-Roach BA, Hatem M, Naik R. Optimal primary surgical treatment for advanced epithelial ovarian cancer. Cochrane Database Syst Rev. 2011;10(8):CD007565.

57. Fujiwara K, Aotani E, Hamano T, et al. A randomized Phase II/III trial of 3 weekly intraperitoneal versus intravenous carboplatin in combination with intravenous weekly dose-dense paclitaxel for newly diagnosed ovarian, fallopian tube and primary peritoneal cancer. Jpn J Clin Oncol. 2011;41:278-282.

58. Fujiwara K. Three ongoing intraperitoneal chemotherapy trials in ovarian cancer. J Gynecol Oncol. 2012;23(2):75-77.

59. Votanopoulous K, Ihemelandu C, Shen P, Stewart J, Russell G, Levine EA. A comparison of hematologic toxicity profiles after heated intraperitoneal chemotherapy with oxaliplatin and mitomycin C. J Surg Res. Epub March 10, 2012. [ahead of print].
Cancer Management and Research

\section{Publish your work in this journal}

Cancer Management and Research is an international, peer-reviewed open access journal focusing on cancer research and the optimal use of preventative and integrated treatment interventions to achieve improved outcomes, enhanced survival and quality of life for the cancer patient. The journal welcomes original research, clinical \& epidemiological

\section{Dovepress}

studies, reviews \& evaluations, guidelines, expert opinion \& commentary, case reports \& extended reports. The manuscript management system is completely online and includes a very quick and fair peerreview system, which is all easy to use. Visit http://www.dovepress.com/ testimonials.php to read real quotes from published authors. 